\title{
Probability Structure of Time Fractional Schrödinger Equation
}

\author{
A. TOFIGHI* \\ Department of Physics, Faculty of Basic Science, University of Mazandaran \\ P.O. Box 47416-1467, Babolsar, Iran
}

(Received July 19, 200\%; in final form July 1, 2009)

\begin{abstract}
We consider the motion of a particle under the influence of a real potential within the framework of time fractional Schrödinger equation in $1+1$ dimensions. For the basis states we obtain a simple expression for the probability current equation. In the limit where the order of fractional derivative is close to unity we find a fluctuating probability density for the basis states. We also provide a compact expression for the time rate of change of the probability density in this case. For the special case where the order of fractional derivative is equal to $\frac{1}{2}$ we compute the probability density.
\end{abstract}

PACS numbers: 03.65.-w

\section{Introduction}

Fractional quantum mechanics is a theory to discuss quantum phenomena in fractal environments. There has been many investigations on the subject. It is formulated as a path integral over Lévy flight [1,2], or from Nottale's scale relativity theory $[3,4]$. An algebraic treatment of the subject is given in $[5,6]$. Finally, a deformed momentum operator is introduced in [7].

The probability structure of space-fractional quantum mechanics is given by

$$
\frac{\partial \rho}{\partial t}+\nabla \cdot \boldsymbol{j}=0,
$$

where $\boldsymbol{j}$ is a fractional probability current density and $\rho=\psi^{*} \psi$ is the usual probability density [2].

In Ref. [8] the Schrödinger equation is considered with the first order time derivative changed to a Caputo fractional derivative. The resulting Hamiltonian is found to be non-Hermitian and time dependent. The structure of probability current equation for the free particle case also has been obtained in [8]. In comparison to the space-fractional quantum mechanics the structure of probability equation is more complicated.

The aim of the present paper is to study this probability structure in detail. In Sect. 2 first we find a general probability current equation for a particle moving under the influence of a real potential. Then by considering the basis states, we find the time rate change of the probability density. In Sections 3 and 4 we examine the case where the order of fractional derivative is close to unity.

\footnotetext{
* e-mail: A.Tofighi@umz.ac.ir
}

Utilizing a powerful expansion method $[9,10]$ we find the wave function and the probability density. We prove that for the basis state the probability density increases initially, then fluctuates around a constant value in later times. We express the time rate of change of this probability density in terms of simple function. We see that in the steady state it fluctuates around zero. Moreover, we prove that the result of the expansion method is identical to the result that can be extracted from the exact solution. In Sect. 5 we discuss the special case of $\alpha=\frac{1}{2}$ and we express the weighted probability density in terms squares of absolute value of a complementary error function. And finally in Sect. 6 we draw our conclusions.

\section{Time fractional Schrödinger equation}

In this modern era and with the advent of many new materials such as polymers, glasses, liquid crystals, biopolymers one would expect that the law and characteristics of motion of a particle in such environments (complex systems) is different from that of a simple system. In fact, such deviations have been observed for variety of classical phenomena [11]. In this work we therefore seek an anomalous relation between energy and the angular frequency of the form

$$
E \propto \omega^{\alpha} \text { where } 0<\alpha<1,
$$

this is called fractional Planck quantum energy relation. This assumption will imply a time fractional Schrödinger equation of the form

$$
\mathrm{i}^{\alpha} \hbar_{\alpha} \frac{\partial^{\alpha} \psi}{\partial t^{\alpha}}=-\frac{\hbar^{2}}{2 m} \frac{\partial^{2} \psi}{\partial x^{2}}+V(x) \psi,
$$

where $\hbar_{\alpha}=M_{\mathrm{P}} c^{2} T_{\mathrm{P}}^{\alpha}$ is a scaled Planck constant. The pa- 
rameters $M_{\mathrm{P}}$ and $T_{\mathrm{P}}$ are Planck mass and Planck time, respectively, and $V(x)$ is a real potential. Next we assume $\psi(x, t)=\varphi(t) \chi(x)$ and an energy relationship of the form $E=\hbar_{\alpha} \omega^{\alpha}$. The unit of $E$ is as usual the unit of energy. The unit of $\hbar_{\alpha}$ is the unit of energy multiplied by $(\mathrm{s})^{\alpha}$ and the unit of $\omega$ is $(s)^{-1}$. From Eq. (3) we obtain

$$
\frac{\mathrm{d}^{\alpha} \varphi(t)}{\mathrm{d} t^{\alpha}}-\frac{\omega^{\alpha}}{\mathrm{i}^{\alpha}} \varphi(t)=0
$$

and

$$
-\frac{\hbar^{2}}{2 m} \frac{\mathrm{d}^{2} \chi(x)}{\mathrm{d} x^{2}}+[V(x)-E] \chi(x)=0 .
$$

There exists a multitude of definitions [9] like RiemannLiouville, Weyl, Reisz, and Caputo for the fractional derivative. However, in this paper we only use the Caputo fractional derivative as it is easier to apply the initial conditions in this type. The left (forward) Caputo fractional derivative for $\alpha>0$ is defined by

$$
\begin{aligned}
\mathrm{D}^{\alpha} f(t) & ={ }_{0}^{\mathrm{C}} \mathrm{D}_{t}^{\alpha} f(t)=\frac{1}{\Gamma(n-\alpha)} \int_{0}^{t} \frac{f^{(n)}(\tau)}{(t-\tau)^{\alpha+1-n}} \mathrm{~d} \tau, \\
n-1 & <\alpha<n, \quad \text { and } t>0,
\end{aligned}
$$

where $n$ is an integer number and $f^{(n)}(\tau)$ denotes the $n$-th derivative of the function $f(\tau)$. It is possible to use an identity for the Caputo derivative of order $0<\alpha<1$ and find $[8]$ :

$$
\frac{\partial \psi}{\partial t}=-\frac{\eta}{\mathrm{i}^{\alpha}} \frac{\partial^{2} \tilde{\psi}}{\partial x^{2}}+\frac{\xi}{\mathrm{i}^{\alpha}} \tilde{\psi}+\frac{\left[\frac{\partial^{\alpha} \psi}{\partial t^{\alpha}}\right]_{t=0}}{t^{1-\alpha} \Gamma(\alpha)},
$$

where

$$
\eta=\frac{\hbar^{2}}{2 m \hbar_{\alpha}}, \quad \xi=\frac{V(x)}{\hbar_{\alpha}} \quad \text { and } \quad \tilde{\psi}=\frac{\partial^{1-\alpha} \psi}{\partial t^{1-\alpha}} .
$$

The discussion of [8] for the probability current equation is only for a free particle. In the case of a particle moving under the influence of a real potential we find

$$
\frac{\partial \rho}{\partial t}+\eta \frac{\partial j}{\partial x}=2 \operatorname{Re}\left[\frac{\eta A+\xi B}{\mathrm{i}^{\alpha}}+\frac{C}{t^{1-\alpha} \Gamma(\alpha)}\right],
$$

where

$$
A=\frac{\partial \tilde{\psi}}{\partial x} \frac{\partial \psi^{*}}{\partial x}, \quad B=\psi^{*} \tilde{\psi}
$$

and

$$
C=\psi^{*}\left[\left(\frac{\partial^{\alpha} \psi}{\partial t^{\alpha}}\right)_{t=0}\right],
$$

the fractional probability current density is given by

$$
j=\frac{\psi^{*}}{\mathrm{i}^{\alpha}} \frac{\partial \tilde{\psi}}{\partial x}+\frac{\psi}{(-\mathrm{i})^{\alpha}} \frac{\partial \tilde{\psi}^{*}}{\partial x},
$$

and the symbol "Re" in Eq. (9) stands for the real part.

The general quantum state for this theory is $\Psi=$ $\sum c_{n} \psi_{n}(x, t)$ and the basis state $\psi_{n}(x, t)$ is given by

$$
\psi_{n}(x, t)=\chi_{n}(x) \varphi_{n}(t) \text {. }
$$

However, it is hard to make any quantitative predictions from the probability current equation for a general quantum state.

So we only consider the basis states. One can show that it is always possible to find real functions for the bound state solutions of Eq. (5). Now from Eq. (4) and the fact that $\chi_{n}(x)$ is a real function we find that $j=0$, moreover the contribution of the first two terms of the right hand side of the probability current equation vanishes as well. Therefore the probability equation in this case is

$$
\frac{\partial \rho_{n}}{\partial t}=2 \operatorname{Re}\left[\frac{C_{n}}{t^{1-\alpha} \Gamma(\alpha)}\right]
$$

where

$$
C_{n}=\psi_{n}^{*}\left[\left(\frac{\partial^{\alpha} \psi_{n}}{\partial t^{\alpha}}\right)_{t=0}\right] .
$$

Now knowing the solution of the $\varphi$ functions are MittagLeffler with a complex argument we find

$$
\frac{\partial \rho_{n}}{\partial t}=2 \operatorname{Re}\left[\frac{\mathrm{D}_{n} E_{\alpha}\left(\left(\mathrm{i} \omega_{n} t\right)^{\alpha}\right)}{t^{1-\alpha} \Gamma(\alpha)}\right],
$$

where the coefficient $\mathrm{D}_{n}$ is given by

$$
\mathrm{D}_{n}=\frac{\omega_{n}^{\alpha} \chi_{n}^{2}(x)}{\mathrm{i}^{\alpha}} .
$$

\section{Limiting case of $\alpha=1-\varepsilon$}

When the order of fractional derivative $\alpha$ is close to unity, namely where $\alpha=1-\varepsilon$, with small $\varepsilon$, then one can use an expansion. The method has been introduced in [9]. The main advantage of this expansion method is that it opens the way for analytical calculations in the realm of fractional calculus.

In this method for the Caputo fractional derivative of order $(1-\varepsilon)$ one defines

$$
\mathrm{D}^{1-\varepsilon} f(t)=f^{(1)}(t)+\varepsilon \mathrm{D}_{1}^{1} f(t)+\ldots,
$$

where $\mathrm{D}_{1}^{1} f(t)$ is

$$
\begin{gathered}
\mathrm{D}_{1}^{1} f(t)=f^{(1)}(0) \ln t+\gamma f^{(1)}(t) \\
+\int_{0}^{t} f^{(2)}(\tau) \ln (t-\tau) \mathrm{d} \tau,
\end{gathered}
$$

where $\gamma$ is the Euler constant. In previous works we have applied this method to the case of fractional oscillation [10] and fractional relaxation phenomena [12].

Essentially one treats the case of low-level fractionality as a perturbation to the integer case. One defines an expansion of the physical variable of interest, namely

$$
\varphi(t)=\varphi_{0}(t)+\varepsilon \varphi_{1}(t)+\ldots
$$

Upon insertion of Eqs. (17)-(19) in Eq. (4) we find

$$
\frac{\mathrm{d} \varphi_{0}}{\mathrm{~d} t}-\frac{\omega}{\mathrm{i}} \varphi_{0}=0,
$$

with solution $\varphi_{0}(t)=\exp (-\mathrm{i} \omega t)$.

For the correction term we get

$$
\frac{\mathrm{d} \varphi_{1}}{\mathrm{~d} t}-\frac{\omega}{\mathrm{i}} \varphi_{1}=-\left(D_{1}^{1}+\frac{\omega}{\mathrm{i}} \ln \frac{\omega}{\mathrm{i}}\right) \varphi_{0},
$$

with the initial condition $\varphi_{1}(0)=0$.

Now we consider the quantity $\mathrm{D}_{1}^{1} \varphi_{0}$. From expression (18) we have

$$
\begin{aligned}
& \mathrm{D}_{1}^{1} \varphi_{0}=\mathrm{D}_{1}^{1} \exp (-\mathrm{i} \omega t)=-\mathrm{i} \omega \ln (t)-\mathrm{i} \omega \exp (-\mathrm{i} \omega t) \\
& -\omega^{2} \int_{0}^{t} \exp (-\mathrm{i} \omega t) \ln (t-\tau) \mathrm{d} \tau
\end{aligned}
$$

If we compute the Laplace transform (LT) of expression 
(22) we obtain

$$
\operatorname{LT}\left[\mathrm{D}_{1}^{1} \varphi_{0}\right]=\frac{\mathrm{i} \omega \ln s}{s+\mathrm{i} \omega} .
$$

Taking the Laplace transform of Eq. (21) and with the help of expression (23) we find

$$
\Phi_{1}(s)=-\frac{\mathrm{i} \omega \ln s}{(s+\mathrm{i} \omega)^{2}}+\frac{\mathrm{i} \omega \ln (-\mathrm{i} \omega)}{(s+\mathrm{i} \omega)^{2}} .
$$

Therefore in the time domain [13] we will have

$$
\begin{aligned}
& \varphi_{1}(t)=\mathrm{i} \omega t \exp (-\mathrm{i} \omega t)\left[E_{i}(\mathrm{i} \omega t)-\ln (\mathrm{i} \omega)\right]-1 \\
& +\exp (-\mathrm{i} \omega t)+\mathrm{i} \omega t \exp (-\mathrm{i} \omega t) \ln (-\mathrm{i} \omega),
\end{aligned}
$$

where $E_{\mathrm{i}}(t)$ is the exponential integral defined by

$$
E_{i}(t)=\int_{-\infty}^{t} \frac{\exp (y)}{y} \mathrm{~d} y .
$$

The solution will take a simpler form in terms of another exponential integral defined by $-E_{1}(z)=E i(-z)-$ i $\pi[14]$, so $\varphi(t)$ is

$$
\begin{aligned}
& \varphi(t)=\exp (-\mathrm{i} \omega t)+\varepsilon[-1+\exp (-\mathrm{i} \omega t) \\
& \left.-\mathrm{i} \omega t \exp (-\mathrm{i} \omega t) E_{1}(-\mathrm{i} \omega t)\right] .
\end{aligned}
$$

Therefore the probability distribution of a stationary state is

$$
\begin{aligned}
& \rho_{n}(x, t)=\psi_{n} \psi_{n}^{*}=\chi_{n}^{2}(x)\{1+2 \varepsilon[-1+\exp (-\mathrm{i} \omega t) \\
& \left.\left.\quad+\omega t\left(\frac{\pi}{2}-\operatorname{Si}(\omega t)\right)\right]\right\},
\end{aligned}
$$

where $\operatorname{Si}(t)$ is the sine-integral function defined by

$$
\operatorname{Si}(t)=\int_{0}^{t} \frac{\sin (x)}{x} \mathrm{~d} x .
$$

In Fig. 1 we draw the parameter $y=\rho_{n}(x, t) / \chi_{n}^{2}(x)$ versus time. As shown in this figure first we have an increase in the value of this parameter followed by fluctuations around the value $1+2 \varepsilon$

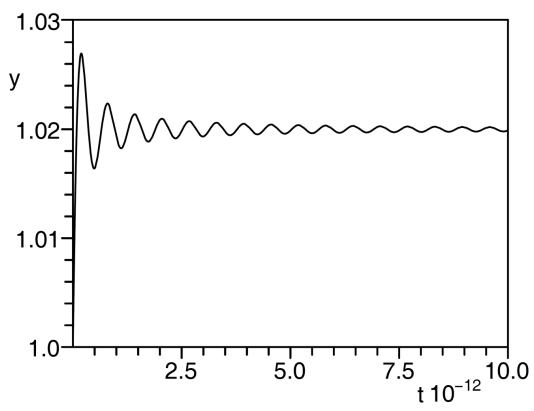

Fig. 1. $y$ versus $t$ for $\varepsilon=0.01$ and $\omega=10^{13}$. The system attains its equilibrium value in few picoseconds, so the basis states are quasi-stationary.

It is useful to evaluate the time rate of change of this probability density. The result is

$$
\frac{\mathrm{d} \rho_{n}(x, t)}{\mathrm{d} t}=-2 \chi_{n}^{2}(x) \varepsilon \operatorname{si}(\omega t),
$$

where $\operatorname{si}(t)$ is the complimentary sine-integral defined by

$$
\operatorname{si}(t)=\operatorname{Si}(t)-\frac{\pi}{2} \text {. }
$$

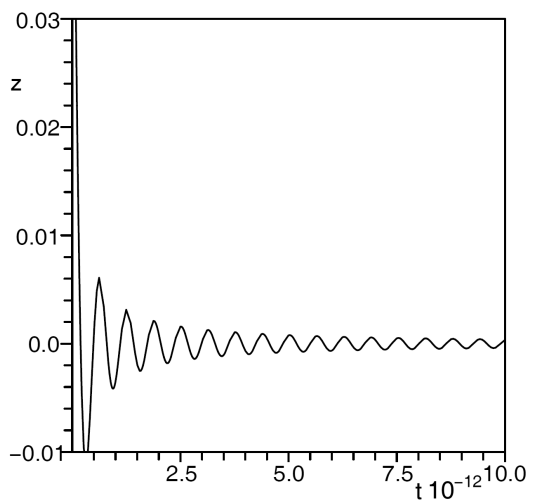

Fig. 2. $z$ versus $t$ for $\varepsilon=0.01$ and $\omega=10^{13}$.

In Fig. 2 we show the variation of the parameter $z=$ $\frac{\mathrm{d} \rho_{n}(x, t)}{\mathrm{d} t} / \chi_{n}^{2}(x)$ versus time.

Here we restrict ourselves to the case of basis states. Moreover, in this section we consider the media with low-level fractionality, where the order of fractional derivative is close to unity. Then it is possible to make quantitative statements about the probability structure of time fractional Schrödinger equation.

We find that in the beginning the source term of the probability current equation creates some particles. But in the fluctuating region whenever the slope of the curve in Fig. 1 is negative it absorbs some particles, and when the slope of the curve is positive it creates particles. This process continues until we reach the steady state when the value of the probability remains approximately constant.

For a value of $\omega=10^{13}$ we see from Fig. 1 that it takes a few picoseconds for the system to attain its equilibrium value. For higher values of frequencies this time will be much shorter. We see that the probability of the basis states are time dependent. But considering the range of energies in ordinary quantum mechanics which is usually of order of a few electron-volts, which corresponds to frequencies of order $10^{16}$, then the main fluctuations which is due to the imposition of fractional derivative occurs within a few millipicoseconds. Therefore the basis states of the theory in the case of a media with low-level fractionality are quasi-stationary. As far as the time rate of change of the probability is concerned, Fig. 2 shows a decreasing function followed by fluctuations around zero. This trend is what is given by Eq. (15) as well.

\section{Comparison with the exact result}

Here we compare the result of our expansion method with the exact calculation. The Mittag-Leffler function with a complex argument can be expressed by [8]:

$$
\begin{aligned}
& E_{\alpha}\left((\mathrm{i} \omega t)^{\alpha}\right)=\frac{\exp (\mathrm{i} \omega t)}{\alpha}-\frac{(\mathrm{i} \omega)^{\alpha} \sin (\alpha \pi)}{\pi} \\
& \quad \times \int_{0}^{\infty} \frac{\exp (-r t) r^{\alpha-1} \mathrm{~d} r}{r^{2 \alpha}-2(\mathrm{i} \omega)^{\alpha} \cos (\alpha \pi) r^{\alpha}+(\mathrm{i} \omega)^{2 \alpha}} .
\end{aligned}
$$


This actually corresponds to $\varphi^{*}(t)$. Now with $\alpha=1-\varepsilon$, and a small $\varepsilon$ we obtain

$$
\begin{gathered}
\varphi^{*}(t)=E_{1-\varepsilon}=\frac{\exp (\mathrm{i} \omega t)}{1-\varepsilon} \\
-\mathrm{i} \omega \varepsilon \int_{0}^{\infty} \frac{\exp (-r t) \mathrm{d} r}{(r+\mathrm{i} \omega)^{2}} .
\end{gathered}
$$

To evaluate the integral of Eq. (33) which we denote by $\lambda$ we make the following change of variable $r^{\prime}=r+\mathrm{i} \omega$ :

$$
\lambda=\exp (\mathrm{i} \omega t) \int_{\mathrm{i} \omega}^{\infty} \frac{\exp \left(-r^{\prime} t\right) \mathrm{d} r^{\prime}}{r^{\prime 2}} .
$$

Using integration by parts and the definition of the exponential integral we find

$$
\lambda=-\exp (\mathrm{i} \omega t)\left[\frac{\mathrm{i}}{\omega} \exp (-\mathrm{i} \omega t)+t E_{1}(\mathrm{i} \omega t)\right] .
$$

Therefore from Eq. (33) we get

$$
\begin{aligned}
& \varphi^{*}(t)=\frac{\exp (\mathrm{i} \omega t)}{1-\varepsilon} \\
& \quad+\mathrm{i} \varepsilon \omega \exp (\mathrm{i} \omega t)\left[\frac{\mathrm{i}}{\omega} \exp (-\mathrm{i} \omega t)+t E_{1}(\mathrm{i} \omega t)\right],
\end{aligned}
$$

which is equal to

$$
\begin{aligned}
& \varphi^{*}(t)=\exp (\mathrm{i} \omega t)+\varepsilon[-1+\exp (\mathrm{i} \omega t) \\
& \left.\quad+\mathrm{i} \omega t \exp (\mathrm{i} \omega t) E_{1}(\mathrm{i} \omega t)\right] .
\end{aligned}
$$

But this is the complex conjugate of expression (27). So the expansion method produces the exact result up to order $\varepsilon$.

\section{The special case of $\alpha=\frac{1}{2}$}

For a general quantum state and a general value of the parameter $\alpha$ it is hard to describe the behavior of the probability density in time fractional Schrödinger equation. It is found [8] that the value of total probability for a free particle increases to the value $\frac{1}{\alpha^{2}}$ in the limit $t \rightarrow \infty$ (assuming the total probability equals unity at $t=0)$.

In this section we discuss the case of $\alpha=\frac{1}{2}$.

The Mittag-Leffler function in this case is [15]:

$$
\begin{aligned}
& E_{\frac{1}{2}}\left(\zeta^{\frac{1}{2}}\right)=\exp (\zeta)\left[1+\operatorname{erf}\left(\zeta^{\frac{1}{2}}\right)\right]=\exp (\zeta) \operatorname{erfc}\left(-\zeta^{\frac{1}{2}}\right), \\
& \quad \zeta \in \mathcal{C},
\end{aligned}
$$

where $\mathcal{C}$ denotes the set of complex numbers and erf (erfc) denotes the (complementary) error function defined as

$$
\begin{array}{r}
\operatorname{erf}(\zeta)=\frac{2}{\sqrt{\pi}} \int_{0}^{\zeta} \exp \left(-u^{2}\right) \mathrm{d} u, \\
\operatorname{erfc}(\zeta)=1-\operatorname{erf}(\zeta), \quad \zeta \in \mathcal{C} .
\end{array}
$$

In Eq. (38) by $\zeta^{\frac{1}{2}}$ we mean the principal value of the square root of $\zeta$ in the complex plane cut along the negative real axis.

For our case

$$
\zeta=-\mathrm{i} \omega t=\exp \left(-\frac{\mathrm{i} \pi}{2}\right) \omega t
$$

as for any complex number $\zeta$ the principal value of $\arg \zeta$ is the unique value of $\arg \zeta$ such that $-\pi<\arg \zeta \leq \pi$. Therefore

$$
\zeta^{\frac{1}{2}}=(-\mathrm{i} \omega t)^{\frac{1}{2}}=\exp \left(\frac{-\mathrm{i} \pi}{4}\right) \sqrt{\omega t}=(1-\mathrm{i}) \sqrt{\frac{\omega t}{2}} .
$$

From Eqs. (38), (40), (41) we obtain the time dependent part of the wave function

$$
\varphi(t)=\exp (-\mathrm{i} \omega t) \operatorname{erfc}[(1-\mathrm{i}) G]
$$

where $G=\sqrt{\frac{\omega t}{2}}$.

The parameter $y$ which is measure of probability density in this case is

$$
y=\frac{\rho_{n}(x, t)}{\chi_{n}^{2}(x)}=|\operatorname{erfc}[(1-\mathrm{i}) G]|^{2} .
$$

In Fig. 3 the parameter $y$ is drawn versus $G$. The probability density starts from unity and then it fluctuates around the value $\frac{1}{\alpha^{2}}$ which is equal to 4 in the present case.

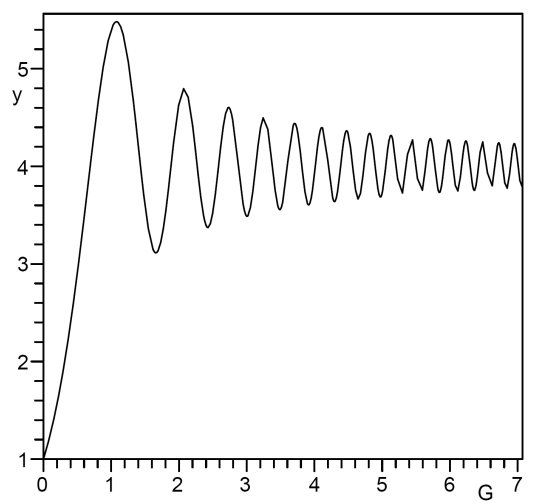

Fig. 3. $y$ versus $G=\sqrt{\omega t / 2}$ for $\alpha=\frac{1}{2}$ and $\omega=10^{13}$.

\section{Concluding remarks}

We have discussed the probability structure of time fractional Schrödinger equation. For the case when the order of fractional derivative is close to unity we find a fluctuating probability density. However, considering the range of energies in ordinary quantum mechanics which is usually of order of a few electron-volts, which corresponds to frequencies of order $10^{16}$, then the main fluctuation which is due to imposition of fractional derivative occurs within a few-millipicoseconds. So the basis states of the theory are quasi-stationary. Furthermore, we see that in the steady state the probability is practically conserved.

For a general value of $\alpha$ like $\alpha=\frac{1}{2}$ we find that the approach to the equilibrium value is much slower than the case of low-level fractionality. Furthermore, the probability is not conserved in this case.

As it has been noted in Ref. [8] there are two ways to fractionalize the time derivative. The first option has been expressed by Eq. (3). The second option is to consider

$$
\mathrm{i} \hbar_{\alpha} \frac{\partial^{\alpha} \psi}{\partial t^{\alpha}}=-\frac{\hbar^{2}}{2 m} \frac{\partial^{2} \psi}{\partial x^{2}}+V(x) \psi .
$$

The wave function and the probability density for a free particle within this option have been studied in $[16,17]$. 
Fractional calculus has been applied in the domain of relativistic field theories as well [17-21]. We hope to study relativistic field theories and possibly unified field theories in the future.

\section{Acknowledgments}

The author thanks Dr. Nozari for helpful discussions. $\mathrm{He}$ is also grateful to the referee for his comments and suggestions.

\section{References}

[1] N. Laskin, Phys. Lett. A 268, 298 (2000).

[2] N. Laskin, Phys. Rev. E 62, 3135 (2000).

[3] L. Nottale, Fractal Space-Time and Microphysics, World Sci., Singapore 1993.

[4] F. Ben Adda, J. Cresson, Chaos, Solitons, Fractals 19, 1323 (2004).

[5] M.A. Lohe, A. Thilagam, J. Phys. A, Math. Gen. 37, 6181 (2004).

[6] M.A. Lohe, Rep. Math. Phys. 57, 131 (2006).

[7] A. Matos-Abiague, J. Phys. A, Math. Gen. 34, 11059 (2001).

[8] M. Naber, J. Math. Phys. 45, 3339 (2004).
[9] V.E. Tarasov, G.M. Zaslavsky, Physica A 368, 399 (2006).

[10] A. Tofighi, H. Nasrolahpour, Physica A 374, 41 (2007).

[11] R. Meltzer, J. Klafter, Phys. Rep. 339, 1 (2000).

[12] A. Tofighi, A. Golestani, Physica A 387, 1807 (2008).

[13] H. Bateman, A. Erdeli, Tables of Integral Transform, Vol. 1, McGraw-Hill, New York 1954.

[14] Handbook of Mathematical Functions with Formulas, Graphs, and Mathematical Tables, Eds. M.A. Abramowitz, I.A. Stegun, 9th ed., Dover, New York 1972

[15] F. Mainardi, R. Gorenflo, J. Comput. Appl. Math. 118, 283 (2000).

[16] M. Bhatti, Int. J. Contemp. Math. Sci. 2, 943 (2007).

[17] M. Bhatti, L. Debnath, Int. J. Pure Appl. Math. 15, 1 (2004)

[18] S.C. Lim, S.V. Muniandy, Phys. Lett. A 324, 396 (2004).

[19] S.C. Lim, Physica A 363, 269 (2006).

[20] E. Goldfain, Chaos, Solitons, Fractals 19, 1023 (2001).

[21] E. Goldfain, Chaos, Solitons, Fractals 28, 913 (2006). 